\title{
Probing the flavor of New Physics with dipoles
}

\section{S. Jäger}

University of Sussex

E-mail: S.Jaegeresussex.ac.uk

\section{K. Leslie}

University of Sussex

E-mail: K.Leslie@sussex.ac.uk

\section{Vale Silva*,}

IFIC, Universitat de València - CSIC

E-mail: luizva@ific.uv.es

Dipole operators encode a rich variety of phenomena, such as radiative decays and electric dipole moments in both quark and lepton sectors, which probe physics beyond the Standard Model up to very high energy scales. Through renormalization, non-dipole operators mix into dipole ones, thus possibly generating observable effects that can be investigated by those same phenomena. I discuss the calculation of this mixing in cases where the leading order effect happens at two-loops (i.e., when one-loop effects vanish), and the phenomenological consequences for flavor and $\mathbf{C P}$ violation coming from New Physics described by operators of dimension six.

European Physical Society Conference on High Energy Physics - EPS-HEP2019 -

10-17 July, 2019

Ghent, Belgium

${ }^{*}$ Speaker. 


\section{Introduction}

Dipole operators encode a rich variety of phenomena, such as radiative decays and Electric Dipole Moments (EDMs) in both quark and lepton sectors, thus probing the Standard Model (SM) structure, and setting bounds on the amount of $\mathbf{C P}$ violation and flavor structure of generic extensions of the SM manifesting in dipoles. Moreover, through renormalization, non-dipole operators mix into dipole ones, then possibly generating observable effects that can be investigated by the same phenomena that probe directly dipole operators.

Given the persistent absence of experimental evidence for non-SM particles below the EW scale, much attention has been given to the systematic study of operators of dimension higher than four involving SM fields only and respecting SM gauged symmetries, suppressed by some power of the characteristic scale of New Physics (NP). In this so-called SM Effective Field Theory (SMEFT) framework, the one-loop Anomalous Dimension Matrix (ADM) of the full set of dim.-six operators was calculated by $[1,2,3]$. The presence of mixing into dipole operators at one-loop sets important bounds on instances of operators of classes $H^{2} X^{2}$ (involving two scalar fields and two field strength tensors), $X^{3}$ (involving three field strength tensors), and $\psi^{4}$ (involving four fermions), cf. e.g. $[3,4]$.

Here, we calculate the mixing into dipoles in some cases where the leading order effect happens at two-loops, i.e., when one-loop ADM elements vanish. Namely, operators of type $\psi^{2} H^{3}$, involving two fermions and three scalars. We then discuss phenomenological bounds on the effective coupling of $\psi^{2} H^{3}$, notably charged lepton radiative decays, and electron and neutron EDMs. While preparing this work, a subset of our results, necessary in the discussion of eEDM, were independently derived in Ref. [5], which we verify.

\section{Basis of operators}

We consider the SM Lagrangian $\mathscr{L}_{\text {SM }}$, added by Right-Handed (RH) neutrinos to enlarge the scope of our analysis, and dimension-six operators, $\mathscr{L}=\mathscr{L}_{\mathrm{SM}+v_{R}}+\sum_{i} C_{i} Q_{i}$, where

$$
\begin{aligned}
\mathscr{L}_{\mathrm{SM}+v_{R}} & =-\frac{1}{4} G_{\mu \nu}^{A} G^{A \mu \nu}-\frac{1}{4} W_{\mu \nu}^{I} W^{I \mu \nu}-\frac{1}{4} B_{\mu v} B^{\mu v}+\left(D_{\mu} H^{\dagger}\right)\left(D^{\mu} H\right)+\sum_{\psi=q, u, d, \ell, e, v_{R}} \bar{\psi} i \not D \psi \\
& -\lambda\left(H^{\dagger} H-\frac{1}{2} v^{2}\right)^{2}-\left[\frac{1}{2}\left(v_{R} C M_{v} v_{R}\right)+H^{\dagger j} \bar{d} Y_{d} q_{j}+\tilde{H}^{\dagger j} \bar{u} Y_{u} q_{j}+H^{\dagger j} \bar{e} Y_{e} \ell_{j}+\tilde{H}^{\dagger j} \bar{v}_{R} Y_{v} \ell_{j}+\text { h.c. }\right]
\end{aligned}
$$

A basis of operators $Q_{i}$ of dimension-six can be found at [6], hereafter called the Warsaw basis, while a basis of operators of dimension-six involving RH neutrinos can be found at, e.g., [7].

To full generality, the renormalization of gauge invariant operators requires, apart from gauge invariant operators themselves (e.g., the operators of the Warsaw basis), referred in the following as physical operators, operators that vanish via EOMs (Equations of Motion), and so-called BRSTexact operators, i.e., operators that are the BRST-variation of another operator; conversely, the renormalization of non-physical operators does not require physical operators as their counterterms $[8,9]$. Operators are non-physical if their matrix elements between physical states vanish [10], and physical otherwise, a property that is then preserved by renormalization. 
When computing the renormalization constants of physical operators in an off-shell scheme, it is fundamental to separate out the effects of other, non-physical classes of operators. It is then necessary to have a basis for these operators, which includes, as an example,

$$
Q_{2-\Gamma}[W] \equiv g_{2} W_{\mu}^{I}\left(\partial_{\nu} \bar{\psi}_{L}\right) \Gamma^{\mu v} \tau^{I}\left(i \not D \psi_{L}\right)-\Sigma_{\xi} Y_{\xi}^{\dagger} g_{2} W_{\mu}^{I}\left(\partial_{\nu} \bar{\psi}_{L}\right) \Gamma^{\mu v} \tau^{I} \xi_{R} \varphi, \quad \Gamma=\eta, \sigma
$$

which is explicitly gauge variant. Note that the last term of $Q_{2-\Gamma}[W]$ defined above carries the same fields of a dipole operator. A basis of non-physical operators will be discussed at [11].

\section{Extraction of the renormalization constants}

Since dimension-six dipole operators have in their field content exactly two fermions, the natural choice of Green's functions to determine the renormalization constants involves two fermions as well, of which we consider those involving as external legs the fields of $\bar{\psi}_{L} \psi_{R} A H, \bar{\psi}_{R} \psi_{L} A H$, $\bar{\psi}_{L} \psi_{L} A, \bar{\psi}_{R} \psi_{R} A, \bar{\psi}_{L} \psi_{R} H, \bar{\psi}_{R} \psi_{L} H, \bar{\psi}_{L} \psi_{L}$, and $\bar{\psi}_{R} \psi_{R}$ (that prove being sufficient in order to extract the renormalization constants of interest), where $A$ is an arbitrary gauge boson field. Solving the set of linear equations resulting from insertions of all possible operators for the renormalization matrix elements describing the mixing among different physical operators, ${ }^{1}$ we have

$$
\begin{aligned}
& Z_{\mathscr{O}, g_{X} \psi^{2} X H} \stackrel{\stackrel{(-)}{M S}}{=} \frac{1}{4 g_{X}}\left\{g _ { X } Q _ { R } ^ { X } \left(-2 \underset{1 / \varepsilon}{\operatorname{Coef}}\left[\mathscr{G}_{\mathscr{O}}\left(\bar{\psi}_{L} \psi_{R} H\right), p^{2}\right]+\underset{1 / \varepsilon}{\operatorname{Coef}}\left[\mathscr{G}_{\mathscr{O}}\left(\bar{\psi}_{L} \psi_{R} H\right), p \cdot p^{\prime}\right]+2 \underset{1 / \varepsilon}{\operatorname{Coef}}\left[\left[\mathscr{G}_{\mathscr{O}}\left(\bar{\psi}_{L} \psi_{R} H\right), \not p p^{\prime}\right]\right)\right.\right. \\
& \left.+g_{X} Q_{L}^{X}\left(-\underset{1 / \varepsilon}{\operatorname{Coef} .\left[\mathscr{G}_{\mathscr{O}}\right.}\left(\bar{\psi}_{L} \psi_{R} H\right), p^{\prime 2}\right]+\underset{1 / \varepsilon}{\operatorname{Coef}}\left[\mathscr{G}_{\mathscr{O}}\left(\bar{\psi}_{L} \psi_{R} H\right), p \cdot p^{\prime}\right]+\underset{1 / \varepsilon}{\operatorname{Coef}}\left[\mathscr{G}_{\mathscr{O}}\left(\bar{\psi}_{L} \psi_{R} H\right), \not p p^{\prime}\right]\right) \\
& \left.+2 \underset{1 / \varepsilon}{\operatorname{Coef}}\left[\mathscr{G}_{\mathscr{O}}\left(\bar{\psi}_{L} \psi_{R} A H\right), k \notin(k)\right]\right\}
\end{aligned}
$$

in the particular case where Green's functions of external legs $\bar{\psi}_{L} \psi_{L} A, \bar{\psi}_{R} \psi_{R} A, \bar{\psi}_{L} \psi_{L}$, and $\bar{\psi}_{R} \psi_{R}$ vanish, where $g_{X}$ are gauge couplings, and $Q_{L}^{X}$ and $Q_{R}^{X}$ are charges of left- and right-handed fields. The kinematics is: $p\left(p^{\prime}\right)$ is the momentum of the incoming (respec., outgoing) fermion, and $k(\varepsilon)$ is the momentum (polarization) of the outgoing gauge boson (the momentum of the outgoing scalar is re-expressed in terms of the other momenta); all momenta are directed as incoming. Note that Green's functions of the kind $\bar{\psi}_{L} \psi_{R} H$ proportional to $\left(p+p^{\prime}\right)^{2}$ do not contribute to $Z_{\mathscr{O}, g_{X} \psi^{2} X H}$.

In the following, we will also give results for the case of $v_{R}$. There are Majorana mass terms that contribute to the EOMs of right-handed neutrinos, and new possibilities for dimension-five operators that also violate leptonic numbers. However, the presence of these terms do not change the relation of the Green's functions and the renormalization constants, since they do not lead to contributions to the Green's functions discussed so far.

We consider dimensional regularization in $D=4-2 \varepsilon$ dimensions. It is possible to state the RGE compactly as

$$
\frac{d C^{T}(\mu)}{d \ell n(\mu)}=-C^{T}(\mu)\left(\frac{d Z}{d \ln (\mu)} Z^{-1}-Z\left(\varepsilon \Delta+\gamma_{M} N\right) Z^{-1}\right) \equiv C^{T}(\mu) \gamma
$$

\footnotetext{
${ }^{1}$ It also results a certain number of equations relating solely Green's functions, that we explicitly verify in our calculations to hold for the $1 / \varepsilon^{2}$ terms.
} 
where $\gamma$ is the ADM, and $\Delta, N$ are diagonal matrices of elements $\delta, n$ : for the dimension-six Yukawa operator $\delta=-3, n=2$; for the dipole $\delta=-1, n=2$. The term proportional to the mass ADM does not contribute to the off-diagonal elements of $\gamma$. Above, $Z_{i j}=\delta_{i j}+\Sigma_{k} \frac{c_{k}}{4 \pi}\left(Z_{k}\right)_{i j}^{(1)}+\ldots$, with $\left(Z_{k}\right)^{(n)}=\sum_{r=0}^{n} \frac{1}{\varepsilon^{r}}\left(Z_{k}\right)_{r}^{(n)}$, where $c_{k}$ denotes a collection of couplings.

The renormalization constant is expanded in the different couplings as follows

$$
\begin{gathered}
Z_{\psi^{2} H^{3}, g_{X} \psi^{2} X H}^{X, \beta \alpha g}=\left[\left(\frac{g_{Y}^{2}}{4 \pi}\left(Z_{Y}^{X}\right)_{1}^{(1)}+\frac{g_{L}^{2}}{4 \pi}\left(Z_{L}^{X}\right)_{1}^{(1)}+\frac{g_{c}^{2}}{4 \pi}\left(Z_{c}^{X}\right)_{1}^{(1)}+\frac{\lambda}{4 \pi}\left(Z_{\lambda}^{X}\right)_{1}^{(1)}+\frac{\Sigma_{Y} \Sigma_{k, l} Y_{k l}^{*} \times Y_{l k}}{4 \pi}\left(Z_{\operatorname{det}^{2}}^{X}\right)_{1}^{(1)}\right) \delta_{f \beta} \delta_{g \alpha}\right. \\
\left.+\frac{\Sigma_{l}\left(Y^{\dagger}\right)_{f l} \times Y_{l \beta}}{4 \pi} \delta_{g \alpha}\left(Z_{y, y}^{X}\right)_{1}^{(1)}+\frac{\left(Y^{\dagger}\right)_{g \beta} \times\left(Y^{\dagger}\right)_{\alpha f}}{4 \pi}\left(Z_{Y, y}^{X}\right)_{1}^{(1)}+\frac{\Sigma_{k} Y_{\alpha k} \times\left(Y^{\dagger}\right)_{k g}}{4 \pi} \delta_{f \beta}\left(Z_{Y, Y}^{X}\right)_{1}^{(1)}\right] \frac{1}{\varepsilon}+\ldots
\end{gathered}
$$

where $\alpha, \beta(f, g)$ designate the flavor indices of $\psi^{2} H^{3}$ (respec., dipole), and the ellipses indicate higher-order terms in the counting of the couplings. At two-loops, the two operators $\psi^{2} H^{3}$ and $\psi^{2} X H$ have the same type of RH field (for instance, $u_{R}$, etc., and not $d_{R}$, etc.), and the indicated Yukawa matrix is the one corresponding to this field (respec., $Y_{u}$, etc.), with the exception of the " $\mathrm{det}^{2}$ " case, that involves all possible Yukawa matrices. There are no other renormalization constants involving different combinations of Yukawa matrices at this order. The results for these renormalization constants are given in Table 1.

\begin{tabular}{cc|cc|cc}
\hline$Q_{\text {phys }}^{L R}[B]$ & $\begin{array}{c}\left(q_{L}, u_{R}\right),\left(q_{L}, d_{R}\right), \\
\left(\ell_{L}, e_{R}\right),\left(\ell_{L}, v_{R}\right)\end{array}$ & $Q_{p h y s}^{L R}[W]$ & $\begin{array}{c}\left(q_{L}, u_{R}\right),\left(q_{L}, d_{R}\right), \\
\left(\ell_{L}, e_{R}\right),\left(\ell_{L}, v_{R}\right)\end{array}$ & $Q_{p h y s}^{L R}[G]$ & $\begin{array}{c}\left(q_{L}, u_{R}\right), \\
\left(q_{L}, d_{R}\right)\end{array}$ \\
\hline$\left(Z_{Y}^{B}\right)_{1}^{(1)}$ & $\frac{3}{4} Q_{\varphi}^{Y} Q_{\varphi}^{Y}\left(Q_{L}^{Y}+Q_{R}^{Y}\right)$ & $\left(Z_{Y}^{W}\right)_{1}^{(1)}$ & $\frac{1}{8} Q_{\phi}^{L} Q_{\varphi}^{Y}\left(Q_{L}^{Y}+Q_{R}^{Y}\right)$ & $\left(Z_{Y}^{G}\right)_{1}^{(1)}$ & 0 \\
\hline$\left(Z_{L}^{B}\right)_{1}^{(1)}$ & $\frac{3}{16} Q_{\varphi}^{Y} Q_{\phi}^{L} Q_{L}^{L}$ & $\left(Z_{L}^{W}\right)_{1}^{(1)}$ & $\frac{3}{32} Q_{\phi}^{L} Q_{\phi}^{L} Q_{L}^{L}$ & $\left(Z_{L}^{G}\right)_{1}^{(1)}$ & 0 \\
\hline$\left(Z_{c}^{B}\right)_{1}^{(1)}$ & 0 & $\left(Z_{c}^{W}\right)_{1}^{(1)}$ & 0 & $\left(Z_{c}^{G}\right)_{1}^{(1)}$ & 0 \\
\hline$\left(Z_{\lambda}^{B}\right)_{1}^{(1)}$ & 0 & $\left(Z_{\lambda}^{W}\right)_{1}^{(1)}$ & 0 & $\left(Z_{\lambda}^{G}\right)_{1}^{(1)}$ & 0 \\
\hline$\left(Z_{y, y}^{B}\right)_{1}^{(1)}$ & $\frac{1}{16}\left(5 Q_{L}^{Y}+Q_{R}^{Y}\right)$ & $\left(Z_{y, y}^{W}\right)_{1}^{(1)}$ & $\frac{1}{32} Q_{L}^{L}$ & $\left(Z_{y, y}^{G}\right)_{1}^{(1)}$ & $\frac{3}{8}$ \\
\hline$\left(Z_{Y, y}^{B}\right)_{1}^{(1)}$ & 0 & $\left(Z_{Y, y}^{W}\right)_{1}^{(1)}$ & 0 & $\left(Z_{Y, y}^{G}\right)_{1}^{(1)}$ & 0 \\
\hline$\left(Z_{Y, Y}^{B}\right)_{1}^{(1)}$ & $\frac{1}{16}\left(Q_{L}^{Y}+5 Q_{R}^{Y}\right)$ & $\left(Z_{Y, Y}^{W}\right)_{1}^{(1)}$ & $\frac{1}{96} Q_{L}^{L}$ & $\left(Z_{Y, Y}^{G}\right)_{1}^{(1)}$ & $\frac{3}{8}$ \\
\hline$\left(Z_{\left.\operatorname{det}^{B}\right)_{1}^{2}}^{(1)}\right.$ & 0 & $\left(Z_{\operatorname{det}^{W}}^{W}\right)_{1}^{(1)}$ & 0 & $\left(Z_{\operatorname{det}^{2}}^{(1)}\right.$ & 0 \\
\hline
\end{tabular}

Table 1: Values are multiplied by $\frac{1}{64 \pi^{3}}$. Above, the dipole operators are $Q_{p h y s}^{L R}[B]=g_{Y} B_{\mu v}\left(\bar{\psi}_{L} \sigma^{\mu v} \xi_{R} \varphi\right)$, $Q_{\text {phys }}^{L R}[W]=g_{L} W_{\mu v}^{I}\left(\bar{\psi}_{L} \sigma^{\mu v} \tau^{I} \xi_{R} \varphi\right), Q_{\text {phys }}^{L R}[G]=g_{c} G_{\mu v}^{a}\left(\bar{\psi}_{L} \sigma^{\mu v} T^{a} \xi_{R} \varphi\right)$, with $\left(\psi_{L}, \xi_{R}\right)$ indicated above, and $\tau^{I}$ $\left(T^{a}\right)$ designating Pauli (Gell-Mann) matrices; $\varphi \rightarrow \phi(\varphi \rightarrow \tilde{\phi})$ for operators involving down-type (respec., up-type) RH fields. Here: $Q_{L}^{L}=Q_{\phi}^{L}=1, Q_{\phi}^{Y}=-Q_{\tilde{\phi}}^{Y}=1 / 2$.

\section{Phenomenology}

Solving Eq. (3.2) at the lowest order we have

$$
C_{g \psi X}^{f g}(\mu)=C_{g \psi X}^{f g}(\Lambda)-2 \times \ln \left(\frac{\Lambda^{2}}{\mu^{2}}\right) \times\left[C_{\psi H}^{f g}(\Lambda) \times\left(\frac{g_{Y}^{2}}{4 \pi}\left(Z_{Y}^{X}\right)_{1}^{(1)}+\frac{g_{L}^{2}}{4 \pi}\left(Z_{L}^{X}\right)_{1}^{(1)}\right)\right]+\ldots
$$


where we omit terms not generated at two-loops and proportional to Yukawas, the ellipses denote further sub-leading terms, and $\mu$ is another (lower) energy scale where the same physical (Warsaw) basis is suitable. The Wilson coefficient $C_{g \psi X}(\Lambda)$ is calculated from the finite part of the twoloop Green's functions involving two fermions, one gauge boson, and one scalar fields. Since no logarithm involving the NP scale is present in $C_{g \psi X}(\Lambda)$, it will count as a sub-leading correction in the counting $\left[\text { coupling }^{2} /(4 \pi)\right]^{m} \times\left[\ln \left(\Lambda^{2} / \mu^{2}\right)\right]^{n}$, which for the term proportional to $C_{\psi H}^{i j}$ in Eq. (4.1) starts at $m=1, n=1$. The calculation of $C_{g \psi X}(\Lambda)$ is beyond the scope of this work.

The Lagrangian describing the vertex $e_{\alpha} e_{\beta} \gamma$ is

$$
\mathscr{L}=\frac{e v}{\sqrt{2}} \mathscr{C}_{e \gamma}^{\beta \alpha} \bar{e}_{\beta} \sigma^{\mu v} P_{R} e_{\alpha} F_{\mu v}+\text { h.c. }
$$

where $\mathscr{C}_{e \gamma}=C_{g e B}-C_{g e W}, \mathscr{C}_{d \gamma}=C_{g d B}-C_{g d W}, \mathscr{C}_{v \gamma}=C_{g v B}+C_{g v W}$, and $\mathscr{C}_{u \gamma}=C_{g u B}+C_{g u W}$ (note that the dipole operators are defined with an overall coupling), see e.g. [12]. ${ }^{2}$ Hereafter, we discuss some phenomenological bounds on the Wilson coefficients of $\psi^{2} H^{3}$, while a more complete phenomenological analysis, including heavy quark transitions, will be discussed in Ref. [11]. For clarity, we first omit important finite contributions (namely, Barr-Zee diagrams), but come back to this point in Section 5.

Higgs couplings. The Lagrangian for Yukawa couplings and fermion mass terms is

$$
\mathscr{L}=-\bar{u} M_{u} u-h \bar{u} Y_{u} u+\ldots, \quad Y_{\psi}=\frac{1}{v_{T}} M_{\psi}\left(1+c_{H, k i n}\right)-\frac{v^{2}}{\sqrt{2}} C_{\psi H}^{\dagger}
$$

where $\psi=u, d, e, v$, the expressions of $c_{H, k i n}=\mathscr{O}\left(\Lambda^{-2}\right)$ and $v_{T} \simeq v$ are given at [3], the mass matrix $M_{\psi}$ is real, and $C_{\psi H}^{\dagger}$ possibly introduces new $\mathbf{C P}$ violating phases and flavor changing neutral couplings. When considering the unitary transformations $u_{L} \rightarrow V_{u} u_{L}, v_{L} \rightarrow U_{v} v_{L}, d_{L} \rightarrow$ $V_{d} d_{L}, e_{L} \rightarrow U_{e} e_{L}$, to move to mass eigenstates, the flavor changing Higgs couplings are given by $\mathscr{L} \ni \frac{v^{2}}{\sqrt{2}} h \bar{u}_{\beta} \tilde{C}_{u H}^{\beta \alpha} u_{\alpha}+\ldots$, where we have defined $\tilde{C}_{u H}=V_{u}^{\dagger} C_{u H}$, etc.; phenomenological bounds will directly probe $\tilde{C}_{u H}$, etc. We consider the leading logarithmic contributions only: in this case, $C_{\xi H}(\mu) \sim C_{\xi H}(\Lambda)$, where $\mu \sim M_{H}$. The experimental constraints on Lepton Flavor Violating (LFV) decays then lead to the following bounds

$$
\begin{aligned}
& \mathscr{B}(h \rightarrow e \mu)<6.1 \times 10^{-5}(95 \% \mathrm{CL})[13] \Rightarrow \sqrt{\left|\tilde{C}_{e H}^{e \mu}\right|^{2}+\left|\tilde{C}_{e H}^{\mu e}\right|^{2}}<5.2 \times 10^{-3} \mathrm{TeV}^{-2} \\
& \mathscr{B}(h \rightarrow e \tau)<4.7 \times 10^{-3}(95 \% \mathrm{CL})[14] \Rightarrow \sqrt{\left|\tilde{C}_{e H}^{e \tau}\right|^{2}+\left|\tilde{C}_{e H}^{\tau e}\right|^{2}}<4.6 \times 10^{-2} \mathrm{TeV}^{-2} \\
& \mathscr{B}(h \rightarrow \mu \tau)<2.8 \times 10^{-3}(95 \% \mathrm{CL})[14] \Rightarrow \sqrt{\left|\tilde{C}_{e H}^{\mu \tau}\right|^{2}+\left|\tilde{C}_{e H}^{\tau \mu}\right|^{2}}<3.6 \times 10^{-2} \mathrm{TeV}^{-2}
\end{aligned}
$$

When setting the bounds above, we have considered $\Gamma_{S M}=4.1 \mathrm{MeV}$ [15], while the experimental bound on the total decay width is $\Gamma<0.013 \mathrm{GeV}$ (95\% CL) [16]. Individually, the partial decay widths of the LFV decays above are below $\sim 0.02 \mathrm{MeV}$.

Radiative decays of charged leptons. The Lagrangian in Eq. (4.2) leads to the following branching ratio for the radiative transition $e_{g} \rightarrow e_{f} \gamma$

\footnotetext{
${ }^{2}$ Tree-level physical amplitudes of a fermion to a massless gauge boson and a fermion are only introduced by dipole operators among the set of dimension-six operators of the Warsaw basis.
} 


$$
\mathscr{B}\left(e_{g} \rightarrow e_{f} \gamma\right)=\alpha \frac{m_{g}^{3}}{\Gamma_{g}} \frac{v^{2}}{2}\left(\left|\mathscr{C}_{e \gamma}^{g f}\right|^{2}+\left|\mathscr{C}_{e \gamma}^{f g}\right|^{2}\right)
$$

where $\mathscr{C}_{e \gamma}^{f g}(\mu)$ is calculated from Eq. (4.1), and contributions proportional to Yukawas are negligible. The bounds from charged lepton radiative decays are the following

$$
\begin{gathered}
\mathscr{B}(\mu \rightarrow e \gamma)<4.2 \times 10^{-13}(90 \% \mathrm{CL})[17] \Rightarrow \sqrt{\left|\tilde{C}_{e H}^{e \mu}(\Lambda)\right|^{2}+\left|\tilde{C}_{e H}^{\mu e}(\Lambda)\right|^{2}} \lesssim 9 \times 10^{-5} \mathrm{TeV}^{-2}=0.1 \times \frac{\sqrt{2 m_{e} m_{\mu}}}{v^{3}} \\
\mathscr{B}(\tau \rightarrow e \gamma)<3.3 \times 10^{-8}(90 \% \mathrm{CL})[18] \Rightarrow \sqrt{\left|\tilde{C}_{e H}^{e \tau}(\Lambda)\right|^{2}+\left|\tilde{C}_{e H}^{\tau e}(\Lambda)\right|^{2}} \lesssim 1 \mathrm{TeV}^{-2} \\
\mathscr{B}(\tau \rightarrow \mu \gamma)<4.4 \times 10^{-8}(90 \% \mathrm{CL})[18] \Rightarrow \sqrt{\left|\tilde{C}_{e H}^{\mu \tau}(\Lambda)\right|^{2}+\left|\tilde{C}_{e H}^{\tau \mu}(\Lambda)\right|^{2}} \lesssim 1 \mathrm{TeV}^{-2}
\end{gathered}
$$

Compared to the bounds derived from LFV Higgs decays, the one from $\mathscr{B}(\mu \rightarrow e \gamma)$ is better by a factor $\sim 60$, while the other two are worse by a factor $\sim 20-30$. For these estimates, we take $\mu$ at $M_{H}$, though the bound on $\mathscr{C}_{e \gamma}$ applies for $\mu \sim m_{\mu}$ : an improvement consists in adopting new effective theories valid at energies (much) below $M_{H}$, cf. e.g. [19], and therefore the bounds shown here carry some extra uncertainties. Similar comments apply for the bounds shown below.

Anomalous Magnetic Moments (AMMs) of charged leptons. The Lagrangian describing the AMM of a fermion $\psi$ is the following

$$
\mathscr{L}_{\mathrm{AMM}}=-\frac{e}{4 m_{\psi}} a_{\psi} \bar{\psi} \sigma^{\mu v} \psi F_{\mu v}
$$

The NP interactions in Eq. (4.2) lead to the correction $\Delta a_{\psi}=-\left(4 m_{\psi} v\right) / \sqrt{2} \times \operatorname{Re}\left[\mathscr{C}_{e \gamma}^{\psi \psi}\right]$ to the SM contribution. The resulting bounds are the following

$$
\begin{aligned}
& \Delta a_{e}=a_{e}^{\exp }-a_{e}^{\mathrm{SM}}=-0.88(0.36) \times 10^{-12}[20] \Rightarrow 0.06 \mathrm{TeV}^{-2} \lesssim \operatorname{Re}\left[\tilde{C}_{e H}^{e e}(\Lambda)\right] \lesssim 0.6 \mathrm{TeV}^{-2} @ 2 \sigma \\
& \Delta a_{\mu}=a_{\mu}^{\exp }-a_{\mu}^{\mathrm{SM}}=268(63)(43) \times 10^{-11}[21] \Rightarrow-7 \mathrm{TeV}^{-2} \lesssim \operatorname{Re}\left[\tilde{C}_{e H}^{\mu \mu}(\Lambda)\right] \lesssim-2 \mathrm{TeV}^{-2} @ 2 \sigma
\end{aligned}
$$

while the AMM of the tau remains largely unconstrained, [21, 22]. Having the values for $\operatorname{Re}\left[\tilde{C}_{e H}^{e e}\right]$ $\left(\operatorname{Re}\left[\tilde{C}_{e H}^{\mu \mu}\right]\right)$ over these ranges translates into mass scales orders of magnitude above the value of $m_{e}$ (respec., $m_{\mu}$ ); in other words, explaining the values of $\Delta a_{e}$ and $\Delta a_{\mu}$ with NP in $\psi^{2} H^{3}$ would require a tuned cancellation among values of the Yukawas introduced by the operators $\psi^{2} H$ and $\psi^{2} H^{3}$.

EDMs of charged leptons. The EDM of a fermion $\psi$ is described by the Lagrangian

$$
\mathscr{L}_{\mathrm{EDM}}=-\frac{i}{2} d_{\psi} \bar{\psi} \sigma^{\mu v} \gamma_{5} \psi F_{\mu \nu}, \quad \frac{d_{\psi}}{e}=-\frac{2 v}{\sqrt{2}} \operatorname{Im}\left[\mathscr{C}_{e \gamma}^{\psi \psi}\right]
$$

The experimental bounds are the following

$$
\left|d_{e}\right| / e<1.1 \times 10^{-29} \mathrm{~cm}(90 \% \mathrm{CL})[23] \Rightarrow\left|\operatorname{Im}\left[\tilde{C}_{e H}^{e e}(\Lambda)\right]\right| \lesssim 2 \times 10^{-7} \mathrm{TeV}^{-2}=0.004 \times \frac{\sqrt{2} m_{e}}{v^{3}}
$$

while the bound for muons [24] and taus [25] translate into NP scales below the EW scale, and thus their treatment based on SMEFT is not meaningful. ${ }^{3}$

nEDM. For the neutron, we have that $d_{N} \sim \frac{1}{3}\left(4 d_{d}-d_{u}\right)$, following from the use of $S U(6)$ quark model in the non-relativistic approximation, see for example [27]. Given Eq. (4.2), we are led to $\left|d_{N}\right|<3.0 \times 10^{-26} e \mathrm{~cm}(90 \% \mathrm{CL})[28] \Rightarrow\left|\operatorname{Im}\left[\tilde{C}_{d H}^{d d}(\Lambda)\right]+1.3 \times \operatorname{Im}\left[\tilde{C}_{u H}^{u u}(\Lambda)\right]\right| \lesssim 4 \times 10^{-3} \mathrm{TeV}^{-2} \sim 9 \times \frac{\sqrt{2} m_{d}}{v^{3}}$

\footnotetext{
${ }^{3}$ For taus, the weak Dipole Moment Ref. [26] could lead to a slightly better, though not yet meaningful, bound.
} 


\section{Discussion and conclusions}

We have discussed how operators of the class $\psi^{2} H^{3}$ can be probed indirectly by their mixinginduced contributions to dipole operators, resulting in a broad set of phenomenogical applications involving both lepton and quark sectors. The leading order mixing of $\psi^{2} \mathrm{H}^{3}$ operators into dipoles at two-loops calculated here leads to important bounds on the Wilson coefficient of $\psi^{2} H^{3}$ derived from $\mathscr{B}(\mu \rightarrow e \gamma)$, eEDM, and nEDM, thus showing the power of dipoles in probing the flavor structure of NP, including the one encoded in non-dipole operators. An improvement of the analysis shown above consists in including contributions from Barr-Zee diagrams, i.e., finite contributions from two-loop insertions of the NP operator $\psi^{2} H^{3}[5,29,30]$, that turn out to be important. When included, the bounds shown previously get improved by a factor of $\sim 2-5$. This feature results from the fact that the ADM elements calculated here, i.e., $\gamma^{X} \sim 1 / 4$, are not large. The dimensionsix operators $\psi^{2} \mathrm{H}^{3}$ are generated in many extensions of the SM involving new heavy degrees of freedom, cf., e.g., [31, 5], that can be therefore constrained by the bounds discussed previously.

In much a similar way, the study presented above can be extended to the mixing of fourfermion operators into dipole operators. At one-loop, $\left(\bar{\ell}^{j} \sigma_{\mu v} e\right) \varepsilon_{j k}\left(\bar{q}^{k} \sigma^{\mu v} u\right)$ is the single operator of the Warsaw basis that mixes ("directly") into dipole operators. At two-loops, a much larger set leads to mixing-induced contributions to dipole processes. It is worth stressing that such contributions can be Yukawa and/or color enhanced. The set of renormalization constants describing the mixing of the whole set of physical four-fermion operators into dipoles, together with their phenomenological analysis, will be presented in [11].

\section{References}

[1] E. E. Jenkins, A. V. Manohar and M. Trott, JHEP 1310, 087 (2013) doi:10.1007/JHEP10(2013)087 [arXiv:1308.2627 [hep-ph]].

[2] E. E. Jenkins, A. V. Manohar and M. Trott, JHEP 1401, 035 (2014) doi:10.1007/JHEP01(2014)035 [arXiv:1310.4838 [hep-ph]].

[3] R. Alonso, E. E. Jenkins, A. V. Manohar and M. Trott, JHEP 1404, 159 (2014) doi:10.1007/JHEP04(2014)159 [arXiv:1312.2014 [hep-ph]].

[4] V. Cirigliano, A. Crivellin, W. Dekens, J. de Vries, M. Hoferichter and E. Mereghetti, Phys. Rev. Lett. 123, no. 5, 051801 (2019) doi:10.1103/PhysRevLett.123.051801 [arXiv:1903.03625 [hep-ph]].

[5] G. Panico, A. Pomarol and M. Riembau, JHEP 1904, 090 (2019) doi:10.1007/JHEP04(2019)090 [arXiv:1810.09413 [hep-ph]].

[6] B. Grzadkowski, M. Iskrzynski, M. Misiak and J. Rosiek, JHEP 1010, 085 (2010) doi:10.1007/JHEP10(2010)085 [arXiv:1008.4884 [hep-ph]].

[7] Y. Liao and X. D. Ma, Phys. Rev. D 96, no. 1, 015012 (2017) doi:10.1103/PhysRevD.96.015012 [arXiv:1612.04527 [hep-ph]].

[8] S. D. Joglekar and B. W. Lee, Annals Phys. 97, 160 (1976). doi:10.1016/0003-4916(76)90225-6

[9] M. Henneaux, Phys. Lett. B 313, 35 (1993) Erratum: [Phys. Lett. B 316, 633 (1993)] doi:10.1016/0370-2693(93)91187-R [hep-th/9306101]. 
[10] J. C. Collins, "Renormalization : An Introduction to Renormalization, The Renormalization Group, and the Operator Product Expansion,” doi:10.1017/CBO9780511622656

[11] S. Jaeger, K. Leslie, L. Vale Silva, in preparation.

[12] A. Crivellin, S. Najjari and J. Rosiek, JHEP 1404, 167 (2014) doi:10.1007/JHEP04(2014)167 [arXiv:1312.0634 [hep-ph]].

[13] G. Aad et al. [ATLAS Collaboration], arXiv:1909.10235 [hep-ex].

[14] G. Aad et al. [ATLAS Collaboration], arXiv:1907.06131 [hep-ex].

[15] A. Denner, S. Heinemeyer, I. Puljak, D. Rebuzzi and M. Spira, Eur. Phys. J. C 71, 1753 (2011) doi:10.1140/epjc/s10052-011-1753-8 [arXiv:1107.5909 [hep-ph]].

[16] V. Khachatryan et al. [CMS Collaboration], JHEP 1609, 051 (2016) doi:10.1007/JHEP09(2016)051 [arXiv:1605.02329 [hep-ex]].

[17] A. M. Baldini et al. [MEG Collaboration], Eur. Phys. J. C 76, no. 8, 434 (2016) doi:10.1140/epjc/s10052-016-4271-x [arXiv:1605.05081 [hep-ex]].

[18] B. Aubert et al. [BaBar Collaboration], Phys. Rev. Lett. 104, 021802 (2010) doi:10.1103/PhysRevLett.104.021802 [arXiv:0908.2381 [hep-ex]].

[19] A. Crivellin, S. Davidson, G. M. Pruna and A. Signer, JHEP 1705, 117 (2017) doi:10.1007/JHEP05(2017)117 [arXiv:1702.03020 [hep-ph]].

[20] R. H. Parker et al. Science 360, 191 (2018) doi:10.1126/science.aap7706

[21] M. Tanabashi et al. [Particle Data Group], Phys. Rev. D 98, no. 3, 030001 (2018). doi:10.1103/PhysRevD.98.030001

[22] S. Eidelman and M. Passera, Mod. Phys. Lett. A 22, 159 (2007) doi:10.1142/S0217732307022694 [hep-ph/0701260].

[23] V. Andreev et al. [ACME Collaboration], Nature 562, no. 7727, 355 (2018). doi:10.1038/s41586-018-0599-8

[24] G. W. Bennett et al. [Muon (g-2) Collaboration], Phys. Rev. D 80, 052008 (2009) doi:10.1103/PhysRevD.80.052008 [arXiv:0811.1207 [hep-ex]].

[25] K. Inami et al. [Belle Collaboration], Phys. Lett. B 551, 16 (2003) doi:10.1016/S0370-2693(02)02984-2 [hep-ex/0210066].

[26] A. Heister et al. [ALEPH Collaboration], Eur. Phys. J. C 30, 291 (2003) doi:10.1140/epjc/s2003-01286-1 [hep-ex/0209066].

[27] M. Pospelov and A. Ritz, Annals Phys. 318, 119 (2005) doi:10.1016/j.aop.2005.04.002 [hep-ph/0504231].

[28] J. M. Pendlebury et al., Phys. Rev. D 92, no. 9, 092003 (2015) doi:10.1103/PhysRevD.92.092003 [arXiv:1509.04411 [hep-ex]].

[29] R. Harnik, J. Kopp and J. Zupan, JHEP 1303, 026 (2013) doi:10.1007/JHEP03(2013)026 [arXiv:1209.1397 [hep-ph]].

[30] J. Brod, U. Haisch and J. Zupan, JHEP 1311, 180 (2013) doi:10.1007/JHEP11(2013)180 [arXiv:1310.1385 [hep-ph]].

[31] S. Davidson, Eur. Phys. J. C 76, no. 5, 258 (2016) doi:10.1140/epjc/s10052-016-4076-y [arXiv:1601.01949 [hep-ph]]. 